\title{
Research Article \\ Comments on Skin Effect in Solitary Solid Tubular Conductor
}

\section{Oldřich Coufal}

Faculty of Electrical Engineering and Communication, Brno University of Technology, Technická 10, 61600 Brno, Czech Republic

Correspondence should be addressed to Oldřich Coufal, coufal@feec.vutbr.cz

Received 3 March 2011; Revised 6 May 2011; Accepted 6 June 2011

Academic Editor: M. Lakshmanan

Copyright (C) 2011 Oldřich Coufal. This is an open access article distributed under the Creative Commons Attribution License, which permits unrestricted use, distribution, and reproduction in any medium, provided the original work is properly cited.

J. C. Maxwell derived formulae for the calculation of current density and current in a cylindrical conductor supplied with variable current. In the 1950s K. Simonyi published a method for calculating current density in a cylindrical conductor made up of two conductors, cylindrical and tubular, of different resistivities. The present paper proves that Simonyi's result is incorrect. The main attention is devoted to the method of calculating current density in a tubular conductor made up of tubular conductors of different resistivities.

\section{Introduction}

Maxwell [1] derived relations for current density and current in a solitary long cylindrical conductor supplied with variable current. In the years 1954-1958, several volumes of an extensive work dealing with the mathematical and physical foundations of electrical engineering were published in Hungary. The author, Simonyi, selected some parts of this work to make a book [2-4], which was published in Germany (ten times, most recently in 1993), USA, and USSR. In [2-4], a method is proposed for the calculation of current density in a cylindrical conductor that is made up of two coaxial solid conductors of different resistivities-a cylindrical and a tubular conductor. In the following, a method is proposed for calculating current density in a tubular conductor made up of two tubular conductors of different resistivities and an analysis is made of the solution of this problem as given in [2-4].

Maxwell's result was the starting point for introducing the concept of skin effect. Current density in the cross-section of a conductor supplied with time-varying current is in fact not constant and increases in the direction towards the conductor surface. The distribution of current density across the conductor cross-section determines the conductor inductance and the forces acting between the solid conductors supplied with varying 


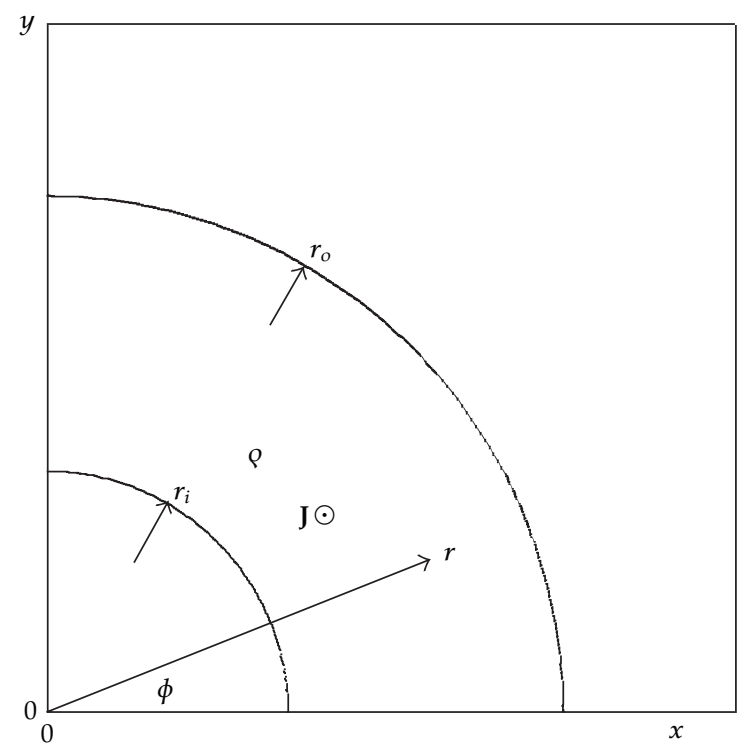

Figure 1: A quarter of the cross-section of tubular conductor.

currents. Current density in an infinitely long straight solid conductor is an approximation of current density in the individual conductors of a system of long straight solid conductors.

\section{Current Density in Long Solitary Tubular Conductor}

Consider a solitary solid tubular conductor of infinite length. The longitudinal axis of the conductor is axis $z$ of a system of Cartesian coordinates $x y z$. In addition to the $x y z$ coordinates the system of cylindrical coordinates $r \phi z$ is also considered. The conductor crosssection in the $x y$ plane is determined by the inequalities $r_{i} \leq r \leq r_{0}, r_{i} \geq 0$ (see Figure 1).

The conductor is connected to an ideal current source $[5,6]$ whose current only depends on $t \in[0, \infty)$ and is sinusoidal, with frequency $f$,

$$
I(t)=\widehat{I} \sin \omega t, \quad \omega=2 \pi f .
$$

The ideal current source supplies current but the current does not flow through the source so that no back conductor to the tube under examination need be considered [7].

The current density vector $\mathbf{J}$ is in the direction of axis $z$ and only depends on $r$ and time $t, \mathbf{J}=(0,0, J)$, with $J=J(r, t)$ having a nonzero value only on the interval $\left[r_{\mathrm{i}}, r_{\mathrm{o}}\right]$. The current flowing through the conductor excites a magnetic field that is determined by the vector $\mathbf{B}$, whose magnitude $B$ also depends on only $r$ and $t$. The fact that $\mathbf{J}, \mathbf{B}$ do not depend on $z$ actually means that an infinitely high propagation velocity of electromagnetic field is assumed. In such a case, the current is considered to be slowly varying [5]. We assume that the permeability of the conductor and its neighbourhood is $\mu_{0}$. Conductor resistivity $\rho=1 / \gamma$, where $\gamma$ is the conductivity, is so low that displacement current $\partial \mathbf{D} / \partial t$ can be neglected. In the whole of this paper, only the part of the conductor between the planes $z=z_{1}$ and $z=z_{2}$, where $z_{1}<z_{2}$ and $z_{2}-z_{1}=z_{21}$, will be considered. 
It has been published many times (most recently in [8]) that the phasor of current density in conductor

$$
\underline{J}(r)=\widehat{J}(r) \exp (\mathrm{j} \alpha(r))
$$

is the solution of the second-order differential equation

$$
\frac{\mathrm{d}^{2} J}{\mathrm{~d} r^{2}}+\frac{1}{r} \frac{\mathrm{d} J}{\mathrm{~d} r}-\mathrm{j} \kappa^{2} J=0, \quad \kappa^{2}=\omega \mu_{0} \gamma
$$

The general solution of (2.3) in the interval $\left[r_{i}, r_{o}\right], r_{i}>0$, depends on two constants $[9,10]$ and is expressed using the Bessel or the Kelvin functions. Of course, the constants cannot be determined from some arbitrary conditions imposed on the solution; in accordance with the theorem of the existence and uniqueness of the solution of $(2.3)[9,10]$, they must be determined from the conditions

$$
\underline{J}(\bar{r})=p, \quad \frac{\mathrm{d}}{\mathrm{d} r} \underline{J}(\bar{r})=d
$$

with $\bar{r}, p$, and $d$ being the given numbers, $\bar{r} \in\left[r_{i}, r_{o}\right]$.

For a cylindrical conductor $\left(r_{i}=0\right),(2.3)$ has a weak singularity [11] at the point $r_{i}=0$. In this case, one of the constants determining the solution is equal to zero, and to determine the other constant the first of conditions (2.4) is sufficient, as was exactly proved in [8]. This fact, together with ignoring the theorem of the existence and uniqueness of the solution of (2.3), has probably led to the belief that the second of conditions (2.4) is superfluous and that the phasor $J$ can be determined by solving (2.3) also for a tubular conductor if $r_{i}>0$. This problem will again be dealt with in Section 3.

\section{Current Density in Composite Conductor}

In the following, the term composite conductor is taken to mean a tubular conductor made up of two coaxial tubular conductors-inner and outer. The inner tubular conductor of a composite conductor is determined by resistivity $\rho_{i}=1 / \gamma_{i}$ and by radii $r_{i 0}, r_{i m}$, with $r_{i 0} \geq$ $0, r_{i 0} \leq r_{i m}$. The outer conductor is determined by resistivity $\rho_{o}=1 / \gamma_{o}$ and radii $r_{o 0}, r_{o n}$, with $r_{i m} \leq r_{o 0}, r_{o 0} \leq r_{o n}$ (see Figure 2). Generally, it could be assumed that the conductor under consideration is made up of an arbitrary number of tubular conductors but solving for two conductors is simpler and also easy to be generalized to an arbitrary number of conductors.

\subsection{Simonyi's Solution}

In [2-4], Simonyi outlined a method for calculating the phasor of electric field intensity $\underline{E}$ in a composite conductor on the assumption that $r_{i 0}=0, r_{i m}=r_{00}$, that is, he solved (2.3) for

$$
\underline{J}=\gamma \underline{E}
$$




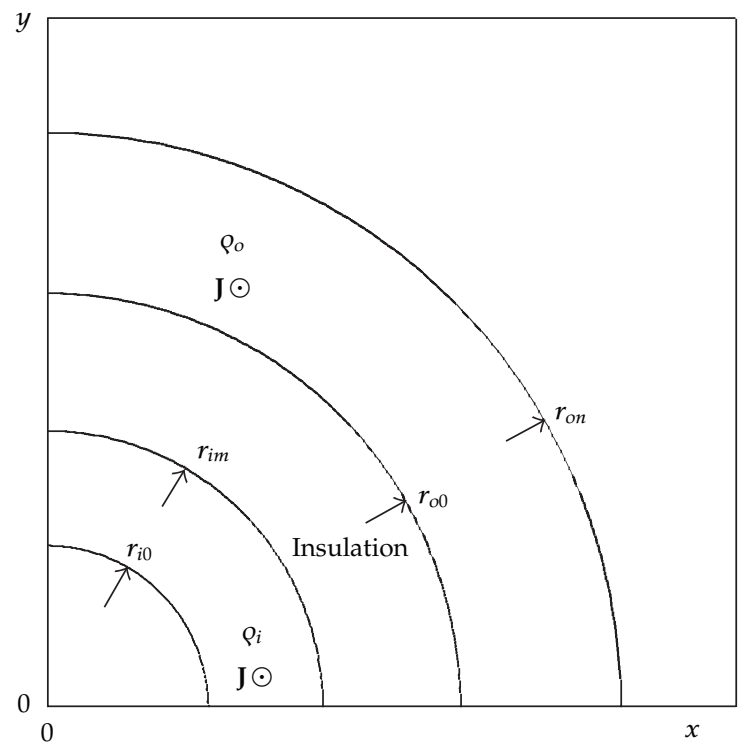

Figure 2: A quarter of the cross-section of composite conductor.

in the inner and the outer conductor on the assumption that the conditions

$$
\begin{aligned}
& \lim _{r \rightarrow r_{i m}-} \underline{E}=\lim _{r \rightarrow r_{i m}+} \underline{E}, \\
& \lim _{r \rightarrow r_{o n}-} \underline{E}=\lim _{r \rightarrow r_{o n}+} \underline{E}, \\
& \lim _{r \rightarrow r_{o^{-}}} \underline{H}=\lim _{r \rightarrow r_{o n}+} \underline{H},
\end{aligned}
$$

are fulfilled. Simonyi's formulation is not absolutely general because in his conception the inner conductor must be cylindrical and there can be no insulation between the conductors (see Figure 2). In [2-4], it is correctly assumed that the sought field $\underline{E}$ depends in the inner conductor on a complex constant $A_{2}$ and in the outer conductor on complex constants $A_{1}$ and $B_{1}$. However, the assertion that these three constants can be established using conditions (3.2), (3.3), and (3.4) is not correct. The calculation of $\underline{A_{1}}, \underline{A_{2}}$, and $\underline{B_{1}}$ was not performed in [2-4].

Simonyi conceives his construction as a determination of electromagnetic field, that is, a determination of fields $\mathbf{E}$ and $\mathbf{H}$ or phasors $\underline{E}$ and $\underline{H}$, with field $\mathbf{J}$ or phasor $J$ being determined by relation (3.1). This complicates the construction unnecessarily and gives the impression that fields $\mathbf{E}$ and $\mathbf{H}$ are to some extent independent. However, the field $\mathbf{E}$ in the conductor and the field $\mathbf{H}$ in the whole space are uniquely determined by the field $\mathbf{J}$ so that the electromagnetic field is determined by the solution of (2.3).

The two conductors forming the composite conductor are solid and homogeneous so that in each conductor the current density will be continuous but there is no reason to assume that it is also continuous for $r=r_{i m}$. There is no reason to assume that vector $\mathbf{E}$ is continuous for $r=r_{i m}$ because relation (3.1) holds. It follows from this that condition (3.2) 
is not generally fulfilled except for the trivial case of $\rho_{i}=\rho_{o}$, which need not be solved as a composite conductor because in [2-4] it is assumed $r_{i m}=r_{o 0}$ (see Example 3.2).

According to [2-4], the value of the limit on the right-hand side of condition (3.3) equals the prescribed electric field outside the conductor. The ideal current source that the conductor is connected to generate an infinitely large voltage on the finite conductor segment considered [6] and thus the electric field outside the conductor cannot be reasonably prescribed.

Condition (3.4) only expresses the fact that the magnetic field is continuous. On the assumption made in the present paper and in [2-4], condition (3.4) is always fulfilled. It is a property of the magnetic field [12], and condition (3.4) is therefore redundant. Conditions (3.2)-(3.4) lack the set value of the derivative $\partial \underline{E} / \partial r$ at some point of the interval $\left[r_{o 0}, r_{o n}\right]$, which is a necessary condition for finding a solution to (2.3) for the electric field. It follows from Simonyi's formulation of the problem and from conditions (3.2) and (3.3) that in the outer conductor, that is, on the interval $\left[r_{00}, r_{o n}\right]$, the electric field can be determined such that it acquires the prescribed values at the end points of this interval. This is not possible since there are infinitely many fields that have the same prescribed value at the initial point $r_{o 0}$ while differing in the values at the end point $r_{o n}$ (see Examples 3.1 and 3.2).

\subsection{Calculation of Current Density in Tubular Conductor}

A method for calculating current density in a solitary solid tubular conductor is described in detail in [8] as model 2. It is proved in [8] that the solution of model 2 leads to the same result as the solution of (2.3). The basic advantage of the method described in [8] is that, to calculate the solution, it is not necessary to know $\partial J / \partial r$. In this subsection, the method for calculating $J$ is described for the inner conductor of a composite conductor.

We choose division $D_{m}$ of the interval $\left[r_{i 0}, r_{i m}\right]$ with the aid of points $r_{i 0}<r_{i 1}<r_{i 2}<$ $\cdots<r_{i m}$. By means of the division $D_{m}$, we can divide the conductor into $m$ partial conductors, with the cross-section of the $k$ th conductor being an annulus determined by the circles $r=$ $r_{i, k-1}$ and $r=r_{i, k}$. The points $r_{i k}, k=1,2, \ldots, m-1$, are chosen such that the partial conductors are of the same cross-section. This means that it holds

$$
a=\pi\left(r_{i, k}^{2}-r_{i, k-1}^{2}\right), \quad \text { where } a=\frac{\pi\left(r_{i m}^{2}-r_{i 0}^{2}\right)}{m}
$$

The conductor resistivity is constant and therefore the current density in the conductor will be continuous with respect to $r$, and thus it can at any instant of time be approximated with arbitrary precision by a function that is constant by parts such that in the $k$ th partial conductor its magnitude is $J_{k}(t)$, independent of $r$. A current $a J_{k}(t)$ flows through the $k$ th partial conductor. The $k$ th and $(k+1)$ th partial conductors between the planes $z=z_{1}$ and $z=z_{2}$ can be replaced by a lumped-elements circuit. For the $k$ th circuit, it holds by Kirchhoff's law

$$
U_{k+1}(t)-U_{k}(t)-U_{L, k}(t)=0, \quad k=1,2, \ldots, n-1,
$$


where $U_{L, k}$ is the voltage induced in the $k$ th circuit and $U_{k}$ and $U_{k+1}$ are voltages across the resistances that substitute the respective partial conductors. The following holds.

$$
U_{k+1}-U_{k}=\rho_{i} z_{21}\left(J_{k+1}-J_{k}\right)
$$

Let us choose the points $c_{1}<c_{2}<\cdots<c_{m}$ such that $r_{i, k-1} \leq c_{k} \leq r_{i, k}$. For a sufficiently large $m$, the electromotive force induced around the boundary of the rectangle $S_{k}=\left[c_{k}, c_{k+1}\right] \times$ $\left[z_{1}, z_{2}\right]$ differs, a little from the voltage $U_{L, k}$ induced in the $k$ th circuit so that using (3.7) and (3.1) (with $\left.\gamma_{i}=1 / \rho_{i}\right)$, (3.6) is in the form

$$
J_{k+1}(t)-J_{k}(t)-\gamma_{i} \sum_{\ell=1}^{m} \Phi_{k \ell} \frac{\mathrm{d}}{\mathrm{d} t} J_{\ell}(t)=0, \quad k=1,2, \ldots, m-1
$$

and at the same time

$$
\Phi_{k \ell}=\int_{c_{k}}^{c_{k+1}} B_{\ell}(r) \mathrm{d} r
$$

where $\boldsymbol{B}_{\ell}(r)$ is magnetic field per unit current density, excited by the $\ell$ th partial conductor.

For a conductor supplied with sinusoidal current, the current density or the complex current density in the $k$ th partial conductor will be

$$
J_{k}(t)=\widehat{J}_{k} \sin \left(\omega t+\alpha_{k}\right)
$$

or

$$
\underline{J_{k}} \exp (\mathrm{j} \omega t), \quad \text { where } \underline{J_{k}}=\widehat{J}_{k} \exp \left(\mathrm{j} \alpha_{k}\right) .
$$

For the complex current density, (3.8) can be rewritten as a system of equations

$$
\underline{J_{k+1}}-\underline{J_{k}}-j \omega \gamma_{i} \sum_{\ell=1}^{m} \Phi_{k \ell} \underline{J_{\ell}}=0, \quad k=1,2, \ldots, m-1
$$

Relation (3.12) is a system of $m-1$ equations for unknown functions $J_{k}(t), k=1,2, \ldots, m$. These functions cannot be determined by means of (3.12) because the number of unknowns is by one greater than the number of equations. System (3.12) can be complemented by adding the equation

$$
a \sum_{\ell=1}^{m} \underline{J_{\ell}}=\underline{I_{i}}
$$

which expresses that the phasor $I_{i}$ of the total current through the conductor is prescribed or by prescribing the value of the current density phasor in a partial conductor, for example, $\underline{J}_{s}$, $1 \leq s \leq m$. 


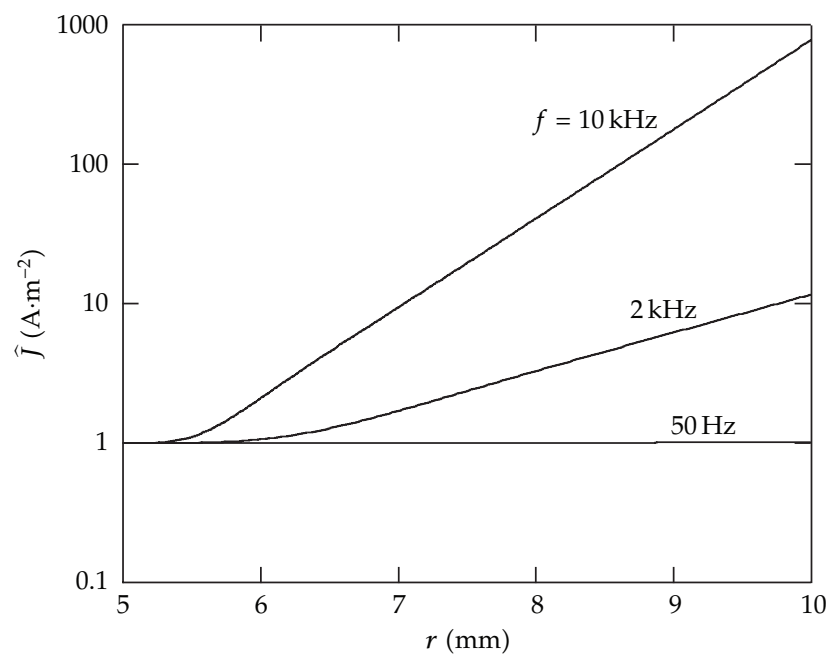

Figure 3: Dependence of current density module on $r$ in Example 3.1; number of partial conductors $m=$ 200.

Example 3.1. A copper tubular conductor has a cross-section given by radii $r_{i}=5 \mathrm{~mm}, r_{o}=10$ $\mathrm{mm}$ (see Figure 1); the value of resistivity at a temperature of $20^{\circ} \mathrm{C} \rho=1.678 \times 10^{-8} \Omega \cdot \mathrm{m}$ has be taken from [13], $f=50,10^{3}$, and $10^{6} \mathrm{~Hz}$, and the value $J\left(r_{i}\right)=1 \mathrm{~A} \cdot \mathrm{m}^{-2} \angle 0^{\circ}$ is given.

The dependence $\widehat{J}(r)$ of the module of current density on $r$ is illustrated in Figure 3. The solution for $f=50 \mathrm{~Hz}$ can be seen in Figure 3 as a constant but $\underline{J}\left(r_{\mathrm{o}}\right)=$ $1.0169 \mathrm{~A} \cdot \mathrm{m}^{-2} \angle 13.48^{\circ}$. The phasor $J$ and the graph of dependence $\widehat{J}(r)$ are determined by the pairs $\left(c_{k}, J_{k}\right)$ for $k=1,2, \ldots, m$. According to [2-4], to solve (2.3) for $J=\gamma \underline{E}$ and a given $f$, it is sufficient to know $\underline{E}\left(r_{i}\right), \underline{E}\left(r_{o}\right)$ and $\underline{H}\left(r_{o}\right)$ (see (3.2)-(3.4)). This means that the solution of (2.3) is given uniquely by the values $J\left(r_{i}\right), J\left(r_{o}\right)$, and $\underline{H}\left(r_{o}\right)$. With the aid of Figure 3 , we can see that for a given $J\left(r_{i}\right)$ there are infinitely many solutions of (2.3) that depend on $f$ (generally on $\kappa$ ), that is, on the value of the derivative $\mathrm{d} J / \mathrm{d} r$ at the point $r_{i}$; this is to say that without knowing the value of this derivative we cannot uniquely determine the solution of (2.3) for a given $f$. It follows from this that until we solve (2.3) for a given $f$ and $J\left(r_{i}\right)$ we cannot know the value of $J\left(r_{o}\right)$. Setting the value $\underline{H}\left(r_{o}\right)$, condition (3.4) not only does not solve this problem, but also in fact counterproductive. The value $\underline{H}\left(r_{o}\right)$ uniquely determines the total current through the conductor and this current can be equal to the calculated current through the conductor only by chance because the current also depends on the set value $J\left(r_{i}\right)$. Conditions (3.2) and (3.4) or (3.3) and (3.4) cannot be set correctly without knowing the solution in the case of tubular conductor.

\subsection{Calculation of Current Density in Composite Conductor}

Let us first consider the case of insulated inner and outer conductors. This means that in the composite conductor there is no circuit formed by the $k$ th partial conductor, which is part of the inner conductor, and by the $(k+1)$ th partial conductor, which is part of the outer conductor. Insulation between the inner and the outer conductor can be of nonzero thickness, that is, $r_{o 0}-r_{i m}>0$, but the case that $r_{i m}=r_{o 0}$, which is solved in [2-4], cannot be excluded. For $r \leq r_{o 0}$, the magnetic field excited by the outer conductor is zero. The outer conductor 
Table 1

\begin{tabular}{lcccc}
\hline Variant & $\rho_{i}\left(10^{-8} \Omega \cdot \mathrm{m}\right)$ & $r_{o 0}(\mathrm{~mm})$ & $r_{\text {on }}(\mathrm{mm})$ & $\rho_{o}\left(10^{-8} \Omega \cdot \mathrm{m}\right)$ \\
\hline 1 & 2.65 & 10 & 15 & 2.65 \\
2 & 2.65 & 10 & 15 & 1.678 \\
3 & 2.65 & 15 & 20 & 2.65 \\
4 & 2.65 & 15 & 20 & 1.678 \\
5 & $10^{8}$ & 10 & 15 & 1.678 \\
6 & $10^{8}$ & 10 & 15 & 1.678 \\
\hline
\end{tabular}

thus cannot affect the inner conductor and therefore current density in the inner conductor is the solution of system (3.12) complemented with the value of phasor $J$ at some point of the inner conductor or with the value of phasor $I_{i}$ in the inner conductor. Naturally, phasor $J$ can be determined by the solution of (2.3), provided we know the numbers $p$ and $d$ in conditions (2.4). The current $I_{i}$ is either set or it can be calculated by means of the current density in the inner conductor, which can be determined prior to calculating the current density in the outer conductor.

We divide the outer conductor into $n$ partial conductors by means of division $D_{n}$, which is determined by the points $r_{o 0}<r_{o 1}<\cdots<r_{o n}$. Similar to the inner conductor, we choose the points $c_{m+1}<c_{m+2}<\cdots<c_{N}, N=m+n, \quad r_{o, \ell-1} \leq c_{m+\ell} \leq r_{o \ell}$. Current density in the outer conductor is the solution of a system of $n$ equations for the phasors $J_{k}, k=m+1, m+2, \ldots, N$. The first $n-1$ equations are (see (3.12)) Kirchhoff's law for the partial circuits:

$$
\underline{J_{k+1}}-\underline{J_{k}}-j \omega \gamma_{0} \sum_{\ell=m+1}^{N} \Phi_{k \ell} \underline{J_{\ell}}=\gamma_{0} \varepsilon_{k}, \quad k=m+1, m+2, \ldots, N-1,
$$

where $\varepsilon_{k}$ is the electromotive force induced in the rectangle $\left[c_{k}, c_{k+1}\right] \times\left[z_{1}, z_{2}\right]$ by time variation of the magnetic flux excited by the inner conductor. The remaining equation is either the prescription of the value of some current density $J_{s}, m<s \leq N$, or the prescription of the phasor of current $\underline{I_{o}}$ in the outer conductor.

Example 3.2. The cross-section of an inner conductor is given by radii $r_{i 0}=5 \mathrm{~mm}, r_{i m}=10 \mathrm{~mm}$; $f=1 \mathrm{kHz}$. The example has 6 variants (see Table 1$) . J\left(r_{00}\right)=1 \mathrm{~A} \cdot \mathrm{m}^{-2} \angle 0^{\circ}$ is always given in the outer conductor. In the inner conductor, in variants $1-5$ it holds $J\left(r_{i m}\right)=J\left(r_{o 0}\right)$, in variant $6 J\left(r_{i m}\right)=0$. Copper resistivity is the same as in Example 3.1; aluminium resistivity $2.65 \times 10^{-\overline{8}} \Omega \cdot \mathrm{m}$ has been taken from [13].

The dependence $\widehat{J}(r)$ of current density module on $r$ in Example 3.2 is illustrated in Figure 4 . Variant 1 is chosen such that it in fact does not concern a composite conductor. The calculation result for the composite conductor in variant 1 is, of course, the same as the calculation result for a tubular conductor. The values $\widehat{J}(r)$ in the inner conductor $\left(r<r_{i m}\right)$ are the same in variants $1-4$ (dotted curve in Figure 4). Using (3.1) to calculate the value $\underline{E}\left(r_{i m}\right)$ and the values $\underline{E}\left(r_{o 0}\right)$ in variants $1-4$, we easily establish that condition (3.2) can but need not be fulfilled.

In [2-4], it is claimed but not proved that by choosing $\gamma_{i}=0$, current density can be determined in the tubular conductor, which in this case is the outer conductor. In other words, the author of [2-4] wanted to say that current density in the outer conductor is the 


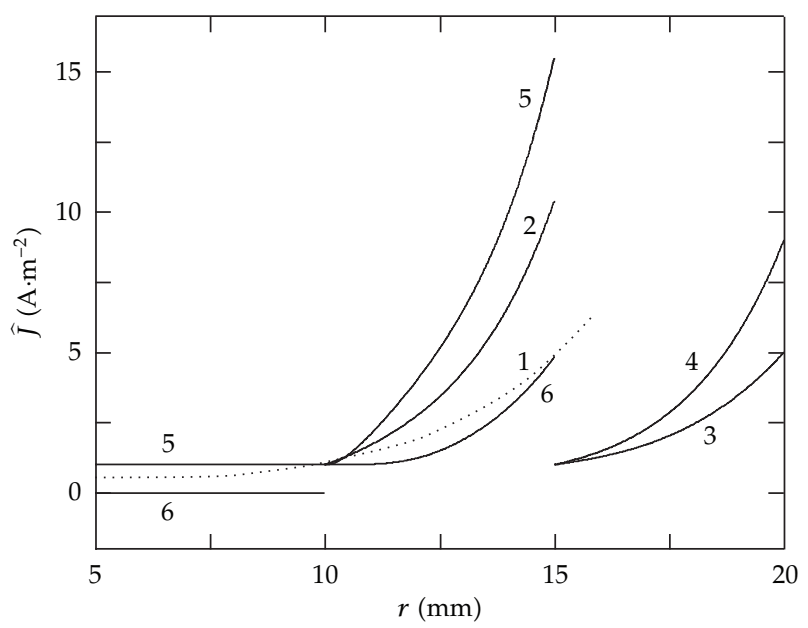

Figure 4: Solution of Example 3.2; variants 1-6 are specified in Table 1; the number of partial conductors in inner and in outer conductor equals 200.

solution of (2.3) and that the conditions necessary for the determination of this solution can be obtained if we assume zero conductivity in the inner cylindrical conductor. In the first place, it is necessary to point out that the condition $\gamma_{i}=0$ does not entail that at any instant of time there must be zero current in the inner conductor. This is easy to verify by solving both (2.3) and (3.12). If current in the inner conductor is nonzero, then the outer conductor is affected by the inner conductor and thus it is not the outer tubular conductor alone that is concerned. If current in the inner conductor is zero, then this information cannot provide conditions (2.4) necessary for the solution of (2.3) in the outer conductor. Variants 5 and 6 of Example 3.2 can complement the above considerations. In both these variants, $\rho_{i}=1 \Omega \cdot \mathrm{m}$ was chosen, which is practically the same as $\gamma_{i}=0$. In variants 5 and 6 , current density in the inner conductor is constant. In variant 5 , unlike variant 6 , current density is nonzero.

There are infinitely many possibilities of choosing two equations that complete system (3.12) in the inner conductor and system (3.14) in the outer conductor. As regards the choice of currents $\underline{I_{i}}$ and $\underline{I_{O}}$, it is necessary to realize that the two currents are completely independent; each conductor is connected to "its" current source. It cannot be assumed that the two conductors (inner and outer) are connected to one current source because the complex impedances $\underline{Z_{i}}$ and $\underline{Z_{o}}$ of the two conductors cannot be determined and thus it is impossible to determine the currents $\underline{I_{i}}$ and $\underline{I_{O}}$ such that it holds

$$
\underline{I_{i}}: \underline{I_{O}}=\underline{Z_{O}}: \underline{Z_{i}}
$$

The phasors $J$ and $\underline{E}$ are connected by relation (3.1). In the present paper, $J$ is preferred. In $[2-4]$, preference is given to the phasor $\underline{E}$, which satisfies condition (3.2) and therefore the following example was chosen.

Example 3.3. Conductor dimensions, frequency $f$ and resistivity values were chosen the same as in variants 1,2, and 6 of Example 3.2. What is different are the prescribed values of phasor 


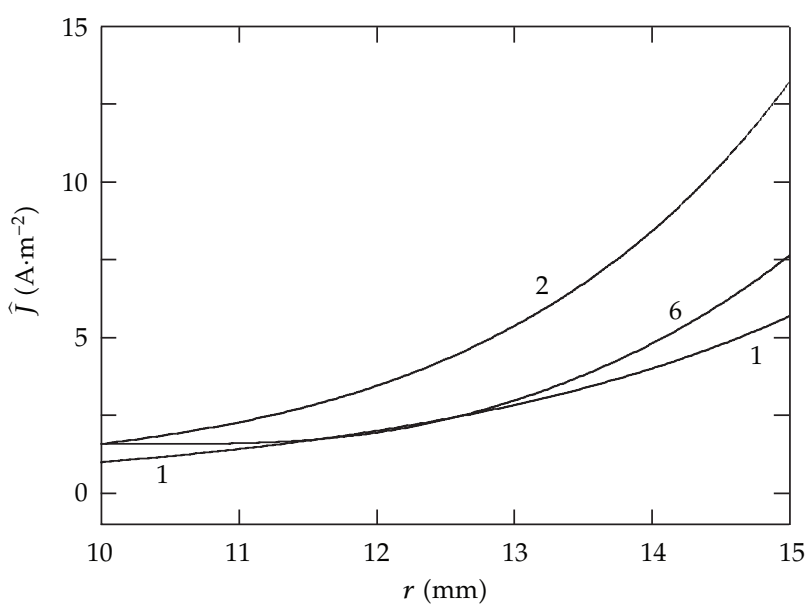

Figure 5: Solution of Example 3.3 in outer conductor. Variants 1, 2 and 6 are specified in the text. The number of partial conductors in inner and in outer conductor equals 200.

$\underline{J}$, which is chosen such that condition (3.2) is satisfied. Phasor $\underline{E}$ is thus assumed to be continuous at the point $r=r_{i m}=r_{00}$, where the prescribed value of $\underline{E}$ is $2.65 \times 10^{-8} \mathrm{~V} \cdot \mathrm{m}^{-1} \angle 0^{\circ}$.

Figure 5 gives the values $\widehat{J}$ of the solution of Example 3.3 in the outer conductor alone. In the inner conductor, these values are the same for variants 1 and 2 and they are the same as for variant 1 in Example 3.2; see Figure 4. By (3.1), in variants 1 and 2 of Example 3.3, $J\left(r_{i m}\right)=1 \mathrm{~A} \cdot \mathrm{m}^{2} \angle 0^{\circ}$ holds, and this is also fulfilled in variant 1 of Example 3.2. The values $\widehat{J}$ in the inner conductor of variant 6 are practically zero since they range from $J\left(r_{i 0}\right)=9.900 \times$ $10^{-9} \mathrm{~A} \cdot \mathrm{m}^{2} \angle-105.57^{\circ}$ to $J\left(r_{i m}\right)=2.650 \times 10^{-8} \mathrm{~A} \cdot \mathrm{m}^{2} \angle 0^{\circ}$. The results of the calculations of phasor $\underline{J}$ lead to the following statement. Let phasor $\underline{E}$ satisfy the condition

$$
\underline{E}\left(r_{i m}\right)=\underline{E}\left(r_{o 0}\right)=\underline{E_{b}},
$$

where $E_{b}$ is the given nonzero complex number. If $\rho_{o}$ is constant, then for $\rho_{i} \rightarrow \infty$ the phasor J converges

(1) in the inner conductor to zero,

(2) in the outer conductor to the current density phasor calculated only in the outer tubular conductor (without the inner conductor) for the given $\underline{E}\left(r_{o 0}\right)=\underline{E_{b}}$.

This statement is an exact expression of what is given in [2-4] as the essence of the method for calculating the phasor $\underline{E}$ or $J$ in tubular conductor. However, the calculation procedure proposed in [2-4] is not correct, as shown in the discussion of the solution of Example 3.2. Moreover, current density in tubular conductor can be determined using a simpler procedure described in Section 3.2. or in [8].

The second case solved in this section is the composite conductor in which the inner and the outer conductor are not mutually insulated. Thus, $r_{i m}=r_{o 0}$ holds and a circuit exists that is formed by the $m$ th partial conductor, which is part of the inner conductor, and by the $(m+1)$ th partial conductor, which is part of the outer conductor. Similar to Example 3.3, we divide the inner conductor into $m$ partial conductors and the outer conductor into $n$ partial 
conductors. In the partial conductors, we choose the points $c_{k}, k=1,2, \ldots, N$. Current density in the composite conductor is determined by the solution of a system of $N$ equations for the phasors $J_{k}, \quad k=1,2, \ldots, N$. The first $m-1$ equations are Kirchhoff's law for the circuits of the inner conductor; these are (3.12). The $m$ th equation is Kirchhoff's law for the common circuit:

$$
\rho_{o} \underline{J_{m+1}}-\rho_{i} \underline{J_{m}}-j \omega \sum_{\ell=1}^{m+1} \Phi_{m \ell} \underline{J_{\ell}}=0
$$

Another $n-1$ equations are Kirchhoff's law for the circuits of the outer conductor; these are the equations

$$
\underline{J_{k+1}}-\underline{J_{k}}-j \omega \gamma_{\mathrm{o}} \sum_{\ell=1}^{N} \Phi_{k \ell} \underline{J_{\ell}}=0, \quad k=m+1, m+2, \ldots, N-1 .
$$

The remaining equation, the $N$ th equation, is either the prescription of the value of some current density $J_{s}, 1 \leq s \leq N$ or the prescription of the phasor of total current $\underline{I}$ in the composite conductor.

Example 3.4. Conductor cross-sections are given by the radii $r_{i 0}=8 \mathrm{~mm}, r_{o n}=11 \mathrm{~mm}$, and $r_{i m}=r_{o 0}$ holds; the inner conductor is of aluminium $\left(\rho_{i}=2.65 \times 10^{-8} \Omega \cdot \mathrm{m}\right)$. For the case a, when $r_{i m}=9 \mathrm{~mm}$, the outer conductor is of copper $\left(\rho_{o}=1.678 \times 10^{-8} \Omega \cdot \mathrm{m}\right), f=0.1 \mathrm{MHz}$; the prescribed value is $J\left(r_{\mathrm{im}}\right)=0.1 \mathrm{~A} \cdot \mathrm{m}^{-2} \angle 0^{\circ}$. For the case $\mathrm{b}$, when $r_{i m}=10 \mathrm{~mm}$, the outer conductor is of lead $\left(\rho_{o}=2.08 \times 10^{-7} \Omega \cdot \mathrm{m}\right), f=1 \mathrm{MHz}$; the prescribed value is $\underline{I}=1 \mathrm{~A} \angle 0^{\circ}$.

The solution of Example 3.4 is given in Figures 6 and 7. Figure 6 gives the dependence of the amplitude $\widehat{J}$ of phasor $J$ on $r$ while Figure 7 gives the dependence of $\alpha=\arg (J)$ on $r$. The lead conductor in the case $\mathrm{b}$ is unusual, and it was chosen only to underline the discontinuity of $\widehat{J}$ on the common border of the inner and the outer conductors. Resistivity of lead at a temperature of $20^{\circ} \mathrm{C}$ has been taken from [13]. The discontinuities in the curves in Figure 7 are only seeming because $\arg (J)$ has been adapted such that its value lies in the interval $(-180,180]$.

Let in the composite conductor in which the inner and the outer conductor are not mutually insulated the current phasor $\underline{I}$ be prescribed. Results of the calculations of phasor $\underline{J}$ lead to the following two statements.

(1) If $\rho_{i}$ is constant, then holds.

$$
\lim _{\rho_{o} \rightarrow \infty} I_{i}=\underline{I}, \quad \lim _{\rho_{o} \rightarrow \infty} I_{o}=0
$$

and current density in the inner conductor converges to the current density calculated only in the inner conductor (without the outer conductor) for the given value $\underline{I_{i}}=\underline{I}$.

(2) If $\rho_{\mathrm{o}}$ is constant, then hold

$$
\lim _{\rho_{i} \rightarrow \infty} I_{\underline{O}}=\underline{I}, \quad \lim _{\rho_{i} \rightarrow \infty} I_{i}=0
$$




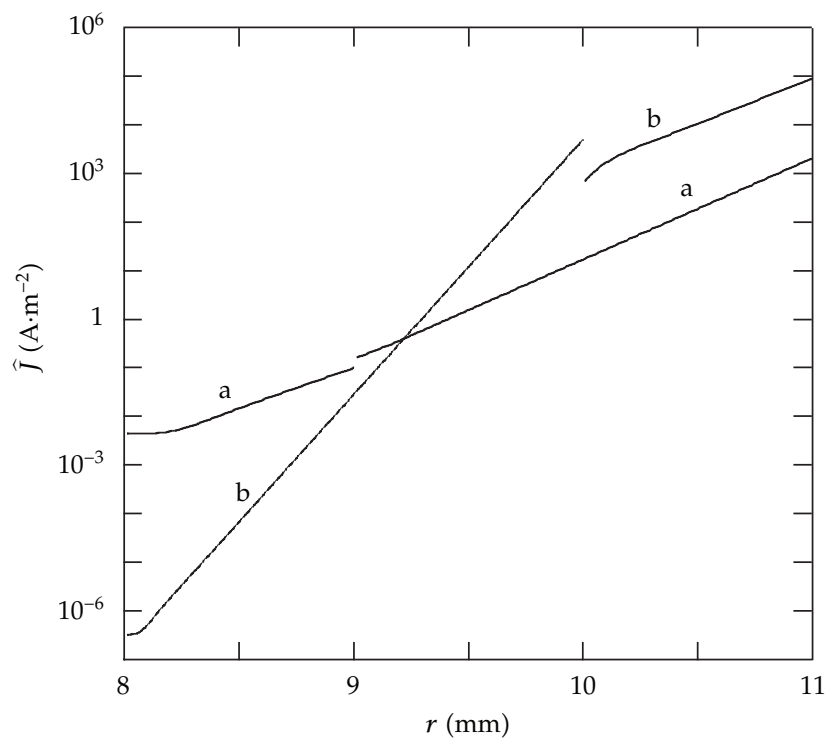

Figure 6: Dependence of the amplitude of phasor $J$ on $r$ in cases a and b of Example 3.4; number of partial conductors $N=250$.

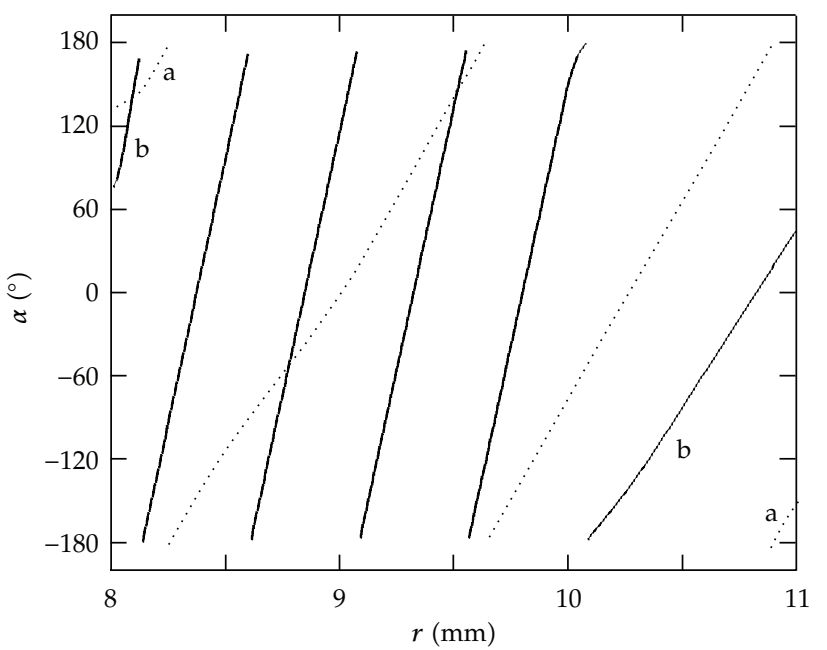

Figure 7: Dependence of $\arg (J)$ on $r$ in cases a (dotted line) and b of Example 3.4; number of partial conductors $N=250$.

and current density in the outer conductor converges to the current density calculated only in the outer conductor (without the inner conductor) for the given value $\underline{I_{O}}=\underline{I}$.

\section{Conclusion}

The calculation of current density in a cylindrical conductor [1] supplied with sinusoidal current is a textbook example that has led to the introduction of the term skin effect and to the calculation of the inductance of cylindrical conductor. In [2-4], Simonyi outlined a method 
for calculating the phasor of electric field intensity in composite conductor. The composite conductor is made up of one cylindrical conductor and one tubular conductor; there is no insulation between the conductors. However, Simonyi did not follow through his proposed method with any resultant formulae and he did not give any solutions of concrete examples either. In the present paper, Simonyi's approach is analyzed and attention is drawn to the fundamental shortcomings of his method.

The main attention is devoted to the method of calculating current density in a tubular conductor made up of tubular conductors of different resistivities. The calculation method is described for the case of a composite conductor made up of two tubular conductors, but the method can easily be generalized for more conductors. In the calculation, it is assumed that the current density phasor in the cross-section of the composite conductor is constant by parts and is the solution of a system of linear equations. The general calculation method is applied in the solution of four examples, each of which has several variants. These examples include several cases. In the first case, the conductor is not a composite conductor but a single tubular conductor. In the remaining cases, the conductor is made up of two tubular conductors, between which there are

(i) insulation of zero thickness,

(ii) insulation of nonzero thickness,

(iii) no insulation.

In the case of nonzero thickness insulation between the conductors, it is worth noting that the skin effect, that is, the increase in current density in the direction towards the conductor surface, manifests itself separately in each conductor of the composite conductor.

\section{Acknowledgment}

This paper contains solution results of the Research Plan no MSM0021630516 of the Ministry of Education, Youth and Sports of the Czech Republic.

\section{References}

[1] J. C. Maxwell, A Treatise on Electricity and Magnetism, vol. 2, Dover Publications, New York, NY, USA, 3rd edition, 1954.

[2] K. Simonyi, Foundations of Electrical Engineering, part 3, VEB, Berlin, Germany, 1956.

[3] K. Simonyi, Foundations of Electrical Engineering, part 3, Pergamon, Oxford, UK, 1963.

[4] K. Simonyi, Foundations of Electrical Engineering, part 3, Mir Publishers, Moscow, Russia, 1964.

[5] J. R. Reitz, F. J. Milford, and R. W. Christy, Foundations of Electromagnetic Theory, chapter 13, AddisonWesley, New York, NY, USA, 1993.

[6] J. Hlávka, Ed., Electrical Engineering I, Physical Foundations, SNTL, Prague, Czech Republic, 1968.

[7] E. N. Miranda, "A simple model for understanding the skin effect," International Journal of Electrical Engineering Education, vol. 36, pp. 31-36, 1999.

[8] O. Coufal, "Current density in a long solitary tubular conductor," Journal of Physics. A, vol. 41, no. 14, Article ID 145401, 2008.

[9] K. Rektorys and E. Vitásek, Eds., Survey of Applicable Mathematics, vol. 1 \& 2 of Mathematics and Its Applications, Kluwer Academic Publishers, Dordrecht, The Netherlands, 2nd edition, 1994.

[10] E. Kamke, Ordinary Differential Equations, chapter 6, Nauka, Moscow, Russia, 1965.

[11] E. Hairer, S. P. Nørsett, and G. Wanner, Solving Ordinary Differential Equations I, Springer, Berlin, Germany, 2nd edition, 1993.

[12] O. D. Kellog, Foundations of Potential Theory, chapter 6, Dover, New York, NY, USA, 1954.

[13] D. R. Lide, Handbook of Chemistry and Physics, CRC Press, Boca Raton, Fla, USA, 88th edition, 2008. 


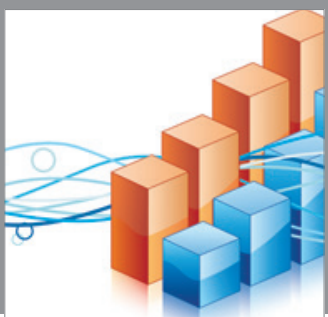

Advances in

Operations Research

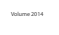

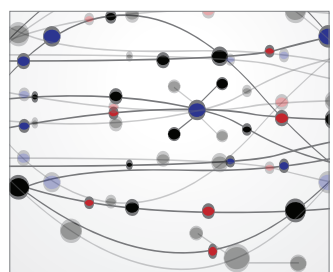

\section{The Scientific} World Journal
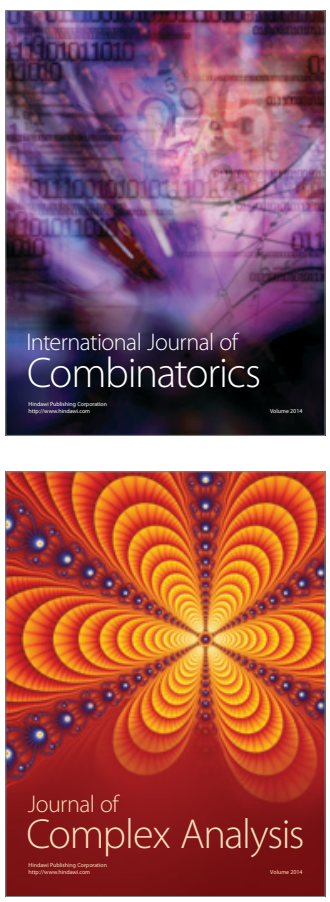

International Journal of

Mathematics and

Mathematical

Sciences
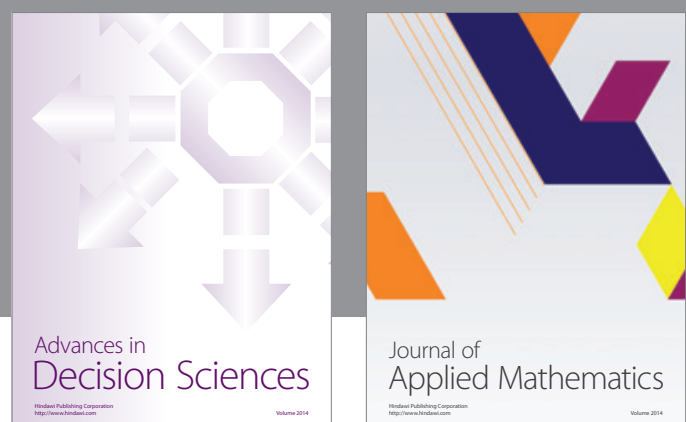

Journal of

Applied Mathematics
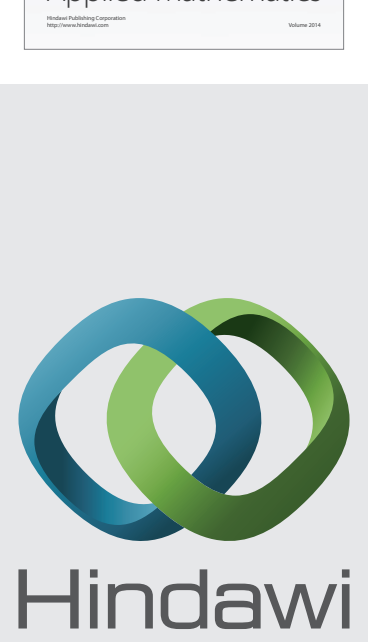

Submit your manuscripts at http://www.hindawi.com
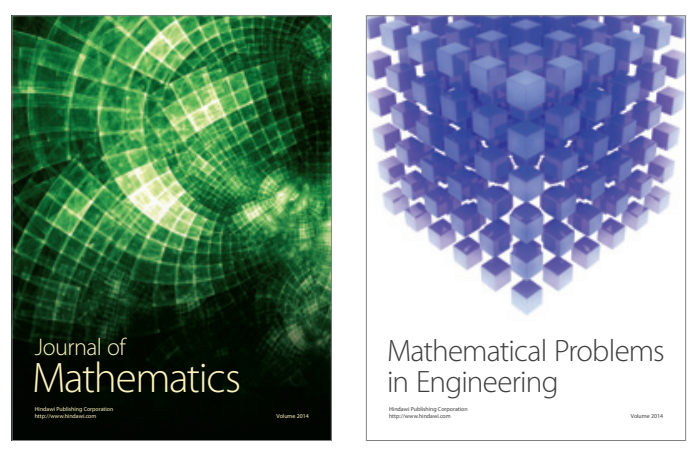

Mathematical Problems in Engineering
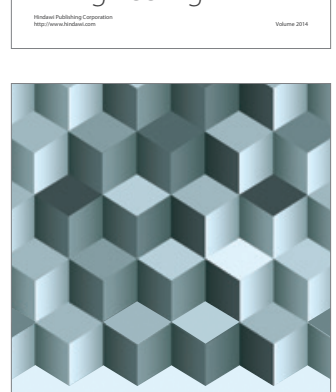

Journal of

Function Spaces
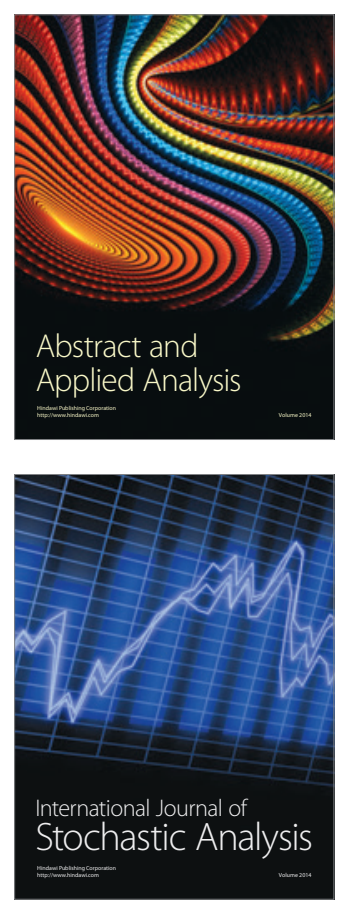

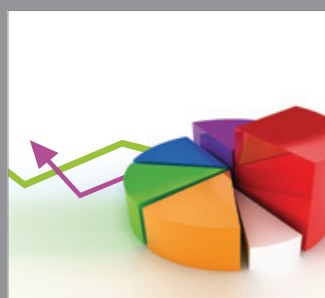

ournal of

Probability and Statistics

Promensencen
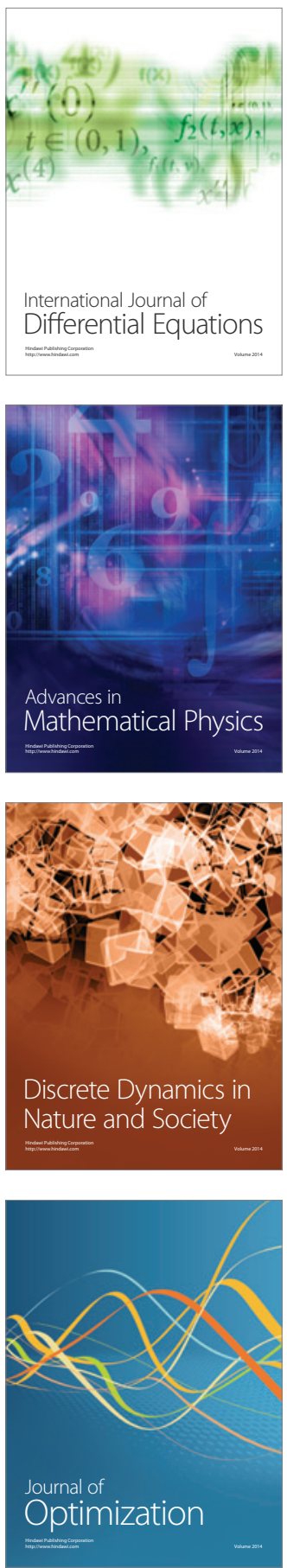